\title{
Cigarette Smoke Exposure Alters Bacterial-Host Interactions in the Respiratory Tract
}

\section{to Promote Disease}

\author{
Pamela Shen, $\mathrm{PhD}^{1,2}$, and Martin R. Stämpfli, $\mathrm{PhD}^{3,4}$
}

${ }^{1}$ Medical Sciences Graduate Program, McMaster University, Hamilton, Ontario, Canada; ${ }^{2}$ Department of Microbiology, New York University, New York,

NY, USA; ${ }^{3}$ Department of Pathology and Molecular Medicine, McMaster Immunology Research Centre, and ${ }^{4}$ Department of Medicine, Firestone Institute

for Respiratory Health at St. Joseph's Healthcare, McMaster University, Hamilton, Ontario, Canada

\section{ABSTRACT}

Epidemiological studies clearly show an increased incidence of respiratory infections and community-acquired pneumonia (CAP) in smokers. Cigarette smoking is also a significant risk factor for invasive pneumococcal diseases (IPDs), leading to meningitis and sepsis. Moreover, the natural course of chronic obstructive pulmonary disease, a spectrum of lung disorders found predominantly in smokers, is punctuated by periods of disease exacerbation caused most frequently by microbial infections. Overall, these infectious episodes contribute to decreased quality of life and mortality, and place a large burden on healthcare systems. In this article, we review how cigarette smoke affects bacterial-host interactions in the upper and lower respiratory tract. We show that smoking affects multiple facets of bacterial-host interactions and postulate that these changes predispose to infection, as smokers fail to control colonizing bacteria in the upper respiratory tract (URT). Hence, we propose that targeting nasal bacterial colonization offers a novel therapeutic avenue to prevent subsequent disease pathogenesis. (BRN Rev. 2017;3:192-203)

Corresponding author: Martin R. Stämpfli, stampfli@mcmaster.ca

Key words: Bacterial colonization. Infection. Inflammation. Smoking. Upper respiratory tract (URT). 


\section{INTRODUCTION}

Cigarette smoking is well known to cause chronic respiratory and cardiovascular disorders, as well as cancer ${ }^{1,2}$. Although less appreciated, the epidemiological evidence for cigarette smoking as a risk factor for various bacterial infections is overwhelming ${ }^{3-9}$. Smoking has long been associated with community-acquired pneumonia (CAP) and significantly increases CAP-associated mortality ${ }^{3,10}$. Moreover, exposure to secondhand smoke (passive smoking) also predisposes to respiratory infections ${ }^{11}$. Additionally, smokers are at increased risk of Legionnaires' disease, tuberculosis, meningococcal disease, otitis media, periodontal disease, and invasive pneumococcal disease (IPD) $)^{4-9}$ (Fig. 1). Notably, the bacterial pathogens associated with these conditions typically enter through the upper respiratory tract (URT) prior to dissemination into the lungs or bloodstream, where disease occurs. While emphasis needs to be placed on reducing smoking prevalence, a greater understanding of mechanisms contributing to disease is equally relevant as the World Health Organization estimates that over one billion of the world's population continue to smoke.

\section{BACTERIAL COLONIZATION OF THE URT}

The URT is colonized by a commensal bacterial microbiome that frequently includes opportunistic pathogens, such as Haemophilus influenza, Moraxella catarrhalis, and Streptococcus pneumoniae, even in healthy individuals ${ }^{12}$. Nasal colonization by opportunistic pathogens is largely asymptomatic, but considered a prerequisite for bacterial invasion into other tissue sites, such as the lungs and blood, where infectious disease onset can occur ${ }^{13}$. Physical and mechanical barriers, such as mucociliary clearance, as well as innate and adaptive host defence mechanisms are critical to the containment and elimination of pathogenic bacterial colonization at the URT mucosa.

Nasal colonization is considered the first step of bacterial dissemination and infectious disease pathogenesis ${ }^{13}$. Smokers are widely reported to have higher incidences of asymptomatic nasal colonization by opportunistic pathogens ${ }^{14-16}$. In a study examining nasopharyngeal bacterial cultures from smokers and non-smokers, increased recovery of pathogens, including S. pneumoniae, $H$. influenzae, $M$. catarrhalis, and S. pyogenes, were observed in smokers ${ }^{14}$. Highly sensitive, global sequencing approaches also confirmed an increase in the presence of potential pathogens such as Staphylococcus aureus, as well as the Streptococcus and Haemophilus bacterial genera in the URT of smokers ${ }^{15,16}$. These observations also extend to passive smoking, which increases pneumococcal carriage ${ }^{17}$. Of note, smoking cessation was associated with reduced occurrences of nasopharyngeal pathogen isolation ${ }^{18}$. As increased incidences of nasal pathogen colonization are associated with higher frequencies of bacterial infection in smokers, several mechanisms underlying increased nasal pathogen acquisition have been investigated (Fig. 2).

\section{MECHANISMS OF INCREASED NASAL PATHOGEN COLONIZATION}

\section{Microbiome dysbiosis}

The human microbiota plays an important role in health and disease. A variety of bacteria 


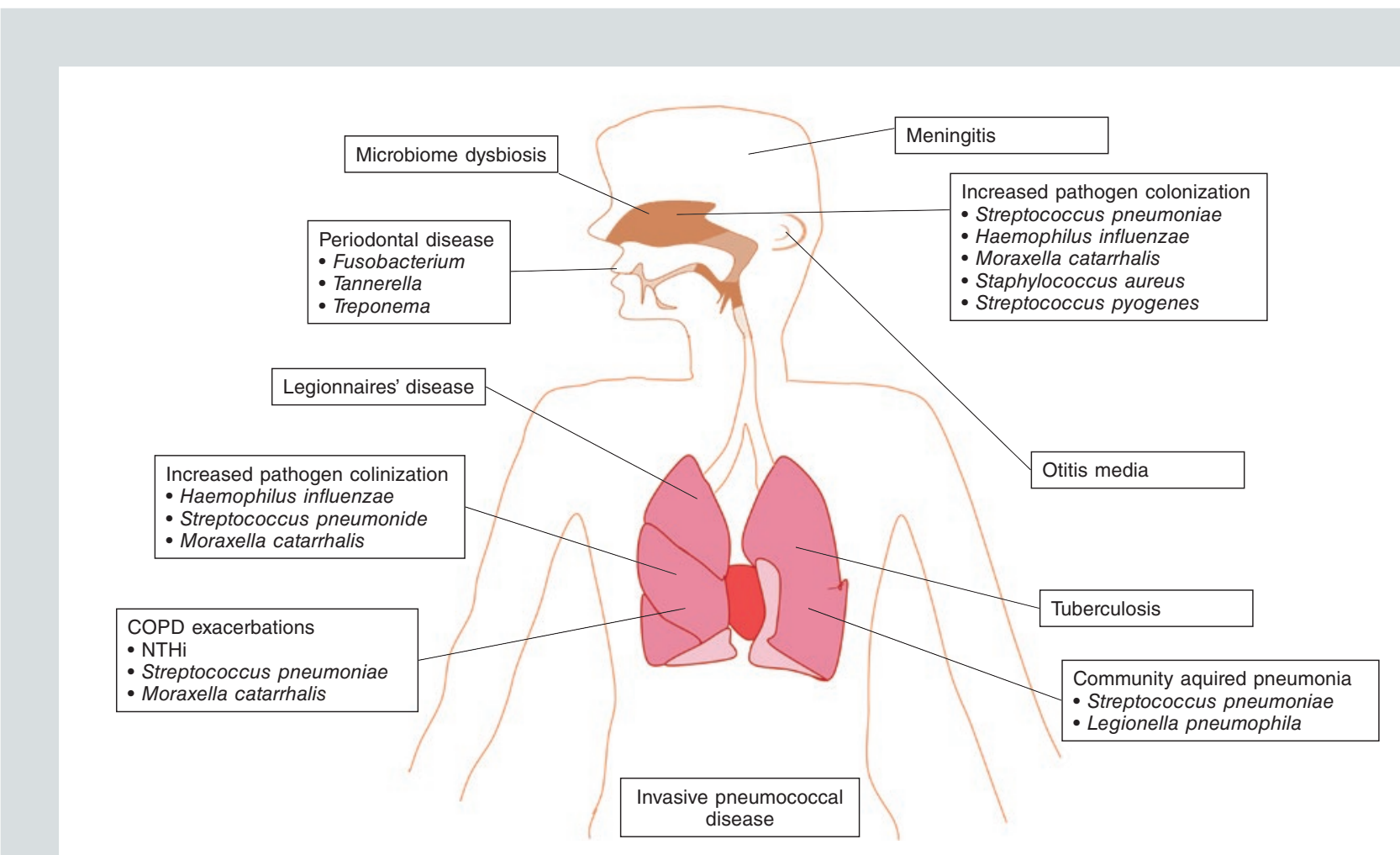

Figure 1. Cigarette smoke exposure predisposes to bacterial infections. Some common pathogens associated with disease are listed. COPD: chronic obstructive pulmonary disease; NTHi: nontypeable Haemophilus influenzae.

colonize the skin, as well as mucosal surfaces of the body including the oral cavity, gut, vagina, and respiratory tract ${ }^{19}$. These microbes react to and influence the microenvironment that they occupy as they interact with each other and the host ${ }^{20}$. The microbiota plays an important role in food metabolism, modulates the host immune system, and, in addition, offers protection from pathogen invasion by competition, termed "colonization resistance" ${ }^{\prime 21}$. Hence, increased pathogen acquisition in the URT of smokers may occur as a result of a perturbation of the nasal microbiota, and compromised colonization resistance ${ }^{14}$.

Smokers are reported to have microbial dysbiosis that could compromise colonization resistance $^{14-16}$. Increased recovery of opportunistic pathogens from the URT of smokers is associated with a decreased incidence of other commensal bacterial isolates, including $\alpha$-hemolytic and non-hemolytic streptococci, as well as Prevotella and Peptostreptococcus capable of competing with pathogen growth ${ }^{14}$. Overall, the resident nasal microbiota of smokers was less able to inhibit pathogenic bacterial growth as compared to that from non-smokers ${ }^{14}$. Sequencing studies found an increased abundance of Firmicutes, including Streptococcus, in both the nasopharynx and the middle meatus of smokers while a reduction in Actinobacteria, such as P. acnes and Corynebacterium was reported ${ }^{15,16}$. Of note, Corynebacterium may play a protective role against the onset of otitis media, and was associated with decreased S. pneumoniae colonization in 

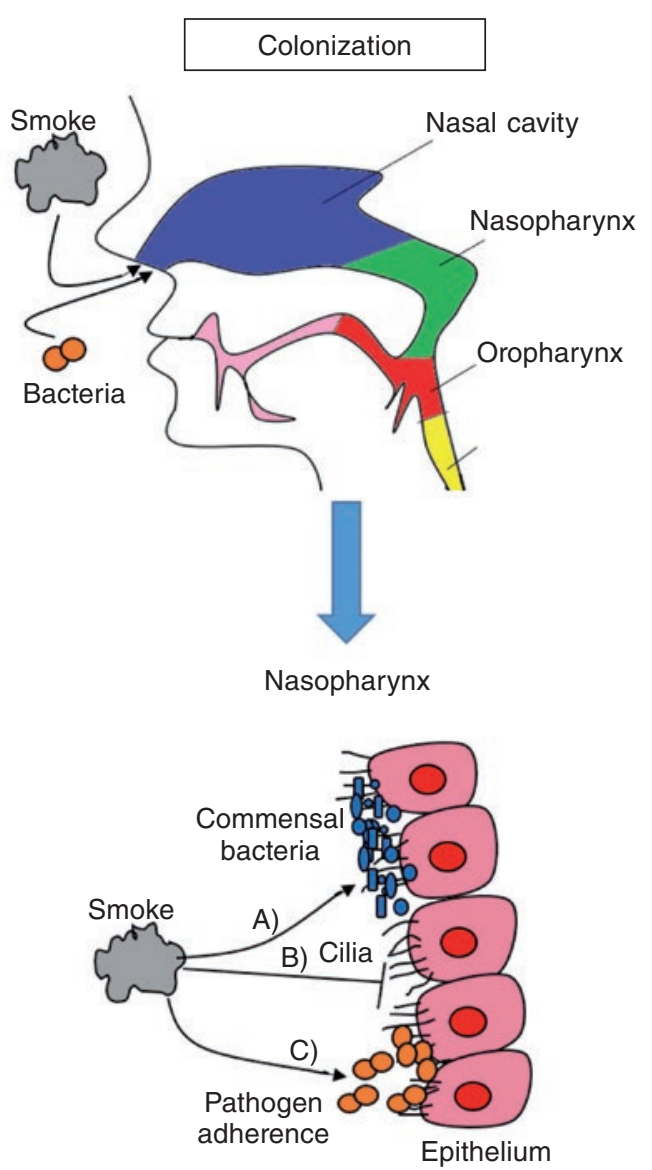

Figure 2. Cigarette smoke exposure increases bacterial pathogen colonization. Smoking increases pathogenic bacterial acquisition and colonization at the upper respiratory tract mucosal surface. This could be due to several mechanisms: A: cigarette smoke alters the microbiome, reducing pathogen colonization resistance; B: cigarette smoke impairs mucociliary clearance; C: smoking facilitates pathogen adherence to host cells.

the URT 22 . Smoking cessation restored the presence of pathogen-interfering nasal bacterial isolates, accompanied by reduced occurrences of nasopharyngeal pathogen isolation $^{18}$. These reports suggest that microbiome dysbiosis and compromised colonization resistance in human smokers contribute to increased pathogen colonization at the URT.
Although highly informative, microbiome studies in human smokers are largely descriptive and cross-sectional. Furthermore, since the URT of smokers already have increased pathogen abundance compared to non-smokers, it is unclear whether microbial dysbiosis (as compared to non-smokers with lower pathogen acquisition) is associated with cigarette smoke exposure alone and present prior to the establishment of pathogen colonization or a consequence of higher nasal pathogen burden. For example, it has been shown that experimental nasal pneumococcal carriage alone in human non-smokers is sufficient to alter the microbiome, with decreased Corynebacterium abundance compared to baseline ${ }^{23}$.

Our laboratory recently investigated the effect of cigarette smoke exposure on the nasal microbiome in the absence and presence of nasal colonization by an opportunistic pathogen, S. pneumoniae, in an experimental mouse model. Although we observed an enrichment in several bacterial genera containing potential pathogens, such as Fusobacterium, Gemella, and Neisseria in smoke-exposed mice, the presence of established nasal pneumococcal colonization was required for these changes in nasal bacterial community to occur (manuscript in submission). Importantly, we showed that cigarette smoke exposure alone was not sufficient to alter the composition of the nasal microbiota. Microbiome composition was also unchanged during early S. pneumoniae colonization in smoke-exposed animals, suggesting that cigarette smoke exposure did not impair the ability of the microbiome to resist initial nasal pneumococcal acquisition. Based on these findings, observations of nasal microbiome dysbiosis in human smokers can be attributed to subsequent disturbance of URT 
microbial communities by established nasal pathogen colonization. Interestingly, we observed that a consequence of established pneumococcal colonization in cigarette smoke-exposed mice was a synergistic interaction between S. pneumoniae and several other potential pathogens, consistent with previous observations of increased pathogen isolation from current smokers ${ }^{14}$. Whether cigarette smoke directly drives synergistic interactions between pathogens following established nasal pneumococcal colonization remains to be determined.

\section{Physical and mechanical barriers}

A critical first line of defence against bacterial infection from colonizing pathogens is the physical barrier of the respiratory tract, consisting of a mucus layer, the respiratory epithelium, goblet mucus cells and basal cells, and the basement membrane ${ }^{24}$. Epithelial cells are physically connected by tight and adherent junctions that prevent pathogen transcellular entry across the epithelium ${ }^{25}$. Cigarette smoke exposure decreases tight junction protein transcription and disrupts cell-to-cell contacts in human epithelial cells, compromising epithelial barrier integrity which may facilitate paracellular pathogen invasion ${ }^{26}$.

Ciliated regions of the airways contain goblet cells and submucosal glands that secrete mucus, a viscous mixture composed of glycoproteins, proteoglycans, lipids, and other proteins ${ }^{27}$. Mucus forms a layer over the epithelium to trap noxious substances, particles and microbes, preventing contact of infectious microbes with the epithelium. The synchronized beating of cilia and mucus production at the airway epithelium together contribute to mucociliary clearance, a critical aspect of the respiratory host defence against microbial infection ${ }^{28}$. The efficiency of mucociliary clearance is dependent on the amount and composition of mucus produced, cilia length and density, as well as ciliary beat frequency ${ }^{27}$. Therefore, any perturbation of mucus production and cilia function may increase the time period of contact between the nasal mucosa and potential infectious microbes, increasing the risk of pathogen colonization and subsequent infection.

Cigarette smoke exposure alters respiratory mucociliary function, compromising the nasal clearance of pathogens. Smokers develop goblet cell hyperplasia and are prone to developing chronic mucus hypersecretion ${ }^{29,30}$. Mucus from smokers are also more dehydrated compared to non-smokers, leading to mucus stasis ${ }^{31}$. In addition, there is a loss of ciliated epithelial cells, and smokers have significantly decreased nasal ciliary beat frequency $^{32}$. Functional tests showed impaired mucociliary clearance in smokers as assessed by saccharin nasal transit time ${ }^{33}$. Thus, mucus stasis induced by cigarette smoking likely facilitates bacterial colonization of the respiratory epithelium and increases the risk of bacterial infections. Indeed, a mouse model of mucus stasis showed increased susceptibility to respiratory tract infection ${ }^{34}$.

Contrary to these reports, we found no significant impact of short term cigarette smoke exposure on either the nasal clearance or bloodstream uptake of ${ }^{99 \mathrm{~m}}$ Technetium-diethylene triaminepentaacetic acid (99mTC-DTPA) following delivery to the upper airways in mice $^{35}$. Clinically, faster clearance of ${ }^{99} \mathrm{~m}$ TC-DTPA through the blood is indicative of compromised epithelial barrier function ${ }^{36}$. Our 
finding implies that acute cigarette smoke exposure did not compromise either nasal clearance or URT epithelial barrier integrity in mice ${ }^{35}$. It may be that longer-term cigarette smoke exposure is required to impact nasal clearance. Along these lines, cigarette smokeexposed mice were reported to have no significant decrease in ciliary beat frequency until after six months of cigarette smoke exposure $^{32}$. However, S. pneumoniae has been observed to both decrease ciliary beat frequency in the human mucosa and down-regulate nasal tight junction protein expression in mice 37,38. $^{3}$. Therefore, it is plausible that the combination of cigarette smoke exposure, even for a short time, and pathogenic bacterial colonization in the upper airways lead to synergistic effects that compromise nasal clearance and epithelial barrier integrity, although this hypothesis has yet to be investigated.

\section{Bacterial adherence}

Cigarette smoke exposure has been reported to increase direct bacterial adherence to host cells, facilitating the establishment of pathogen colonization $^{39,40}$. For example, S. pneumoniae is known to bind several host receptors including the platelet activating factor receptor (PAFR) and polymeric immunoglobulin receptor $(\mathrm{pIgR})^{41,42}$. PAFR is increased in the lungs of smokers and cigarette smoke-exposed mice ${ }^{43}$. S. pneumoniae interacts with the PAFR via a phosphorylcholine moiety (ChoP) that mimics the molecular structure of platelet activating factor, the endogenous ligand to $\mathrm{PAFR}^{44}$. After adherence, S. pneumoniae has been shown to invade host cells via the PAFR following cellular activation by several cytokines, including interleukin-1 alpha (IL-1 $\alpha$ ), and IL-1 administration to rabbits increased lung pneumococcal burden in a PAFR-dependent manner ${ }^{42}$. Pre-treatment with a PAFR antagonist also attenuated lung infection with ChoP-containing strains of Pseudomonas aeruginosa in mice $^{45}$. Additionally, both heart microlesion formation and translocation across the bloodbrain barrier by S. pneumoniae was PAFR dependent in mice ${ }^{46,47}$. We recently showed that cigarette smoke exposure predisposed to IPD in mice independent of both PAFR and IL-1 $\alpha$. Although PAFR may still be potentially involved in pneumococcal dissemination from the lungs following cigarette smoke exposure, our data suggest it played no significant role in promoting invasive disease following nasal colonization ${ }^{35}$. Interestingly, direct translocation of bacteria into human nasal epithelial cells has also been shown to be dependent on $\mathrm{pIgR}^{41}$. Smokers and early chronic obstructive pulmonary disease (COPD) patients have been reported to have higher lung $\mathrm{PIgR}$ lev$\mathrm{els}^{48}$. However, others reported downregulation in COPD patients ${ }^{49}$. Overall, although PAFR is involved in lung infection and IPD, it played little part in URT bacterial dissemination. There is also contradictory data regarding pIgR expression levels in smokers and COPD patients. Whether cigarette smoke exposure increases bacterial binding to these host receptors, thus contributing to colonization and invasive disease remains to be investigated.

\section{BACTERIAL DISSEMINATION FROM THE URT}

Bacteria found in the lungs resemble that of the URT, suggesting microbes are constantly being introduced to the lungs from URT 


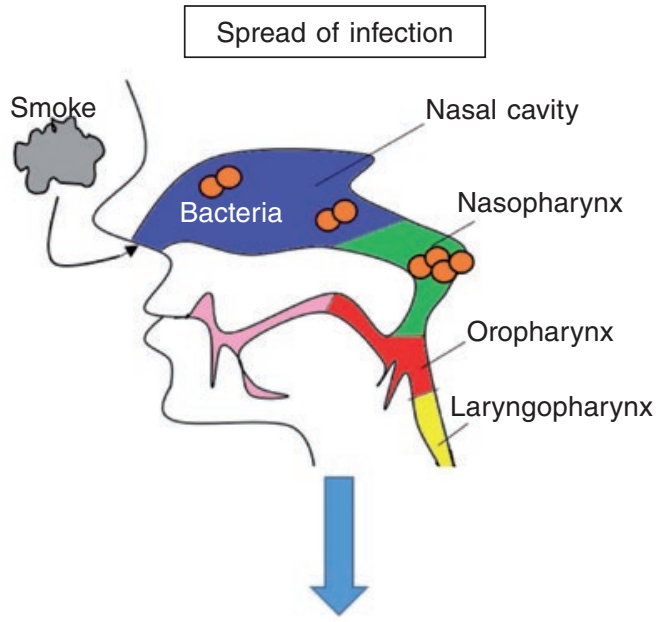

Nasopharynx

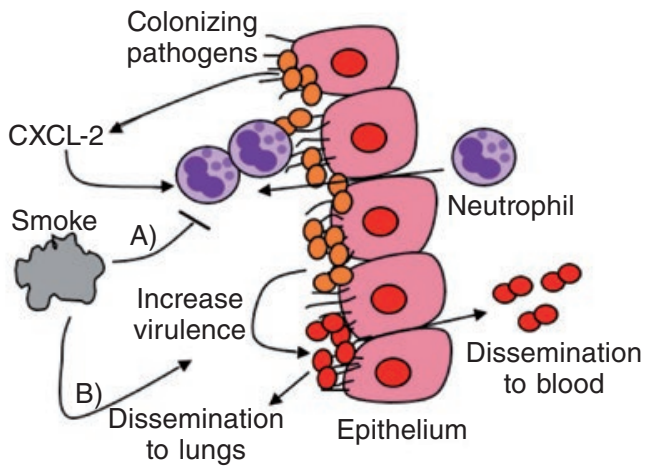

Figure 3. Cigarette smoke exposure predisposes to bacterial dissemination. Following established nasal colonization by pathogenic bacteria, smokers are at increased risk of infections associated with $\mathbf{A}$ : an attenuated nasal host inflammatory response to bacteria and $\mathbf{B}$ : enhanced bacterial virulence. CXCL-2: chemokine (CXC motif) ligand 2.

colonization $^{50}$. Not surprisingly, the increased incidence of lung colonization by opportunistic bacteria found in smokers is mirrored by higher recovery rates of the same bacteria colonizing their nasopharyn $x^{14}$. It is plausible that bacterial-host interactions are altered in smokers, compromising the containment of bacteria in the URT and predisposing to CAP and IPD. Along these lines, we have shown that cigarette smoke exposure predisposed mice to IPD following nasal pneumococcal colonization $^{35}$. This mirrored clinical observations that smokers are over-represented in a population of IPD patients at $58 \%$, as compared to $24 \%$ of control healthy individuals ${ }^{8}$. Alarmingly, even passive smoke exposure increases invasive disease in children and adults $^{8,51}$. Similar to bacterial colonization, we have shown that the effect of smoking is reversible as smoking cessation rescued mice from IPD ${ }^{35}$. Mechanisms of bacterial dissemination are currently not well understood. Clinically, dissemination likely occurs via the lung, as occult bacteraemia is relatively rare (2.8\% of emergency department bacteraemia cases) ${ }^{52}$. Overall, these observations suggest that following nasal pathogen acquisition, cigarette smoke exposure increases the risk of bacterial dissemination and disease (Fig. 3).

\section{MECHANISMS OF INCREASED BACTERIAL DISSEMINATION FROM NASAL COLONIZATION}

\section{The URT host response to bacteria}

In the URT of naive mice, a rapid influx of neutrophils is induced by $S$. pneumoniae, followed by gradual and sustained recruitment of monocytes/macrophages important in resolving colonization ${ }^{53,54}$. Although neutrophils do not directly reduce bacterial burden, they play a role in antigen priming of adaptive immunity that impacts later Th17-dependent macrophage-mediated clearance of colonizing pneumococci ${ }^{53,54}$. Importantly, we observed an impaired ability of cigarette smoke-exposed mice to recruit neutrophils in response to pneumococcal colonization ${ }^{35}$. Specifically, 
cigarette smoke exposure attenuated nasal pro-inflammatory mediator expression, including tumor necrosis factor (TNF)- $\alpha$, chemokine (CXC-motif) ligand (CXCL) -1 and CXCL-2. Strikingly, the effect of cigarette smoke is similar to targeted neutrophil depletion, which had no immediate impact on nasal bacterial burden, but predisposed mice to IPD ${ }^{54}$. Cigarette smoke may thus predispose to IPD by impairing the neutrophilic response induced by nasal pneumococcal colonization. We also reported delayed nasal bacterial clearance following cigarette smoke exposure in mice, consistent with reports of increased incidences of pneumococcal nasal colonization in adolescents exposed to passive smoke ${ }^{17,35}$. Thus, another consequence of an attenuated nasal host response is prolonged nasal colonization, increasing the time period in which individuals are at risk of developing IPD. Restoring inflammatory responses to pathogenic bacteria in the URT is a potential therapeutic approach to decreasing the rate of infection in smokers.

\section{Cigarette smoke and bacterial virulence}

Enhanced bacterial virulence is another potential contributing factor for increased bacterial dissemination from the URT of smokers. In particular, cigarette smoke has been observed to affect biofilm formation in several bacterial species. Adherence to the host epithelium and the establishment of a biofilm is believed to be required for successful nasal colonization of the host ${ }^{55-57}$. This is demonstrated by impaired nasopharyngeal colonization in mice inoculated with biofilm-defective mutant pneumococcal strains ${ }^{55}$. The biofilm state offers several advantages for microbial survival, as demonstrated by greater antibiotic tolerance, a stable protective environment, and shorter bacterial replication time. The biofilm also acts as a reservoir from which large populations of the bacteria can disseminate into new host tissues ${ }^{58}$.

Cigarette smoke exposure has been described to affect microbial biofilm formation, adherence, and gene expression ${ }^{59-62}$. P. aeruginosa, S. pneumoniae, and $S$. aureus biofilm formation was enhanced following cigarette smoke exposure in vitro $59,61,62$. Genes related to detoxification, oxidative stress, DNA repair, and virulence in Porphyromonas gingivalis were increased in response to cigarette smoke extract $(\mathrm{CSE})^{60}$. CSE was also shown to enhance S. aureus adherence to host cells and virulence factor expression ${ }^{61}$. These reports of altered microbial gene expression and biofilm formation may explain the increased risk of bacterial infections in smokers. However, a limitation of these studies is the use of in vitro systems, which may lack physiological relevance $^{59-62}$. Validation of cigarette smoke's effects on microbial virulence in humans and animal models of disease is warranted.

\section{CIGARETTE SMOKE ALTERS THE LUNG BACTERIAL HOST RESPONSE}

Similarly to observations in the URT, cigarette smoking facilitates lung bacterial colonization. $42 \%$ of otherwise asymptomatic smokers were found to be culture positive for potentially pathogenic bacteria, such as $H$. influenzae, $S$. pneumoniae, and M. catarrhalis, while nonsmokers were all culture negative ${ }^{63}$. Moreover, the presence of these pathogens was 
associated with increased levels of TNF- $\alpha$, IL-8, and increased percentages of neutrophils in the bronchoalveolar lavage (BAL) flu$\mathrm{id}^{63}$. It is plausible that the increased presence of bacterial pathogens in the lungs is a reflection of increased dissemination from the URT, but impaired lung mucociliary clearance and attenuated alveolar macrophage (AM) responses are also likely contributing factors. Cigarette smoke-induced mucus hypersecretion and airway plugging may trap aspirated bacteria in the lungs ${ }^{64}$. Bacterial persistence could also be due to a decreased ability of human AMs to phagocytose and mount appropriate cytokine responses to bacterial pathogens ${ }^{65,66}$. These altered lung host responses are also thought to promote pneumonia pathogenesis in smokers ${ }^{11}$.

Contrasting findings in the URT, cigarette smoke exposure is associated with an exaggerated lung host response to bacterial infection, characterized by exacerbated inflammation and increased lung damage in both humans and mice ${ }^{63,67,68}$. It is plausible that chronic and repeated exposure to pathogenic bacteria in smokers contributes to a decline in lung function. In support of this hypothesis, we showed that repeated nontypeable Haemophilus influenzae (NTHi) lung infection in the context of continuous cigarette smoke exposure led to increased lung compliance and airspace enlargement in mice ${ }^{68}$. It has been proposed that this vicious circle of bacterial colonization, inflammation, and lung damage likely contributes to the pathogenesis of COPD (reviewed in detail in Sethi $S$ et al. $\left.{ }^{69}\right)$. The vicious circle hypothesis may also apply to passive smoke exposure, which is associated with lung inflammation, respiratory infections, and COPD development ${ }^{11,70}$.
Importantly, smoking cessation is considered an effective therapeutic treatment for $\mathrm{COPD}^{71}$. Experimentally, we showed that 4 weeks of smoking cessation in mice restored AM TNF- $\alpha$ expression in response to lipopolysaccharides (LPS), suggesting that altered lung host responses may be reversible upon smoking cessation for a sufficient period of time ${ }^{72}$. Further research is warranted to elucidate how smoking alters the lung host response to bacterial infection and contributes to COPD development.

Mechanistically, cigarette smoke exposure induces neutrophil recruitment into the lungs dependent on IL-1 receptor (IL-1R)-1 signal$\operatorname{ling}^{73}$. Activation of the IL-1R1 leads to myeloid differentiation primary response gene 88 (MyD88)-dependent signalling and promotes pro-inflammatory gene transcription through nuclear factor kappa-light-chain-enhancer of activated B cells (NF- $\mathrm{B}$ ) and mitogen-activated protein kinase (MAPK) activation. Both IL-1 $\alpha$ and IL-1 $\beta$, can activate IL-1R1. Precursor IL-1 $\alpha$ is biologically active as a $31 \mathrm{kDa}$ protein. In contrast, IL-1 $\beta$ is initially synthesized as pro-IL-1 $\beta$ (also $31 \mathrm{kDa}$ ), which is biologically inactive and requires proteolytic cleavage by the cysteine protease caspase- 1 for its activation. Caspase- 1 is, in turn, activated by the inflammasome, a multi-protein complex consisting of a member of the Nod-like receptor (NLR) family and the adaptor protein apoptosis-associated speck-like protein (ASC) ${ }^{74}$. We showed cigarette smoke-induced inflammation to be IL-1 $\alpha$, but not IL-1 $\beta$ dependent in mice ${ }^{73}$. Furthermore, chimeric mouse studies suggest that AMs are the cellular source of IL- $1 \alpha$, which activates IL-1R1 within the structural cell compartment, likely the epithelium, to release neutrophil-recruiting chemokines in response to 
cigarette smoke ${ }^{68,73}$. This IL-1 $\alpha$ response in AMs from smoke-exposed mice is further amplified in response to NTHi stimulation, and drives exacerbated neutrophilia in vivo. Downstream of IL-1R1 signalling, exacerbated neutrophilia correlated with levels of CXCL5, and was CXCR2-dependent ${ }^{68}$. How cigarette smoke exposure elicits AMs to produce IL-1 $\alpha$ remain unclear. However, we have reported a potential role for damaged lipids, specifically oxidized low-density lipoproteins (OxLDL) uptake by AMs, in inducing this process ${ }^{75}$. Thus, cigarette smoke exposure elicits an IL-1 $\alpha$ response in the lungs, which is further exacerbated by bacterial infection, leading to excessive neutrophilia associated with lung damage (Fig. 4). This altered lung response may underlie the development of COPD, as well as bacterial exacerbations of disease.

\section{CONCLUSIONS}

Cigarette smoke alters bacterial-host interactions in the respiratory tract to promote disease. Smokers have increased frequencies of acquiring URT pathogenic bacteria. This may be attributable to the impact of cigarette smoke on bacterial-bacterial interactions within the microbiome; specifically, cigarette smoke exposure may provide advantages for synergistic interactions between colonizing pathogens. Other contributing factors to increased URT pathogen acquisition include impaired mucociliary clearance, and increased bacterial adhesion to host cells. Once colonization is established, smokers are predisposed to bacterial dissemination from the URT. In mice, the increased risk of IPD following cigarette smoke exposure is associated with attenuated nasal inflammatory responses normally induced by

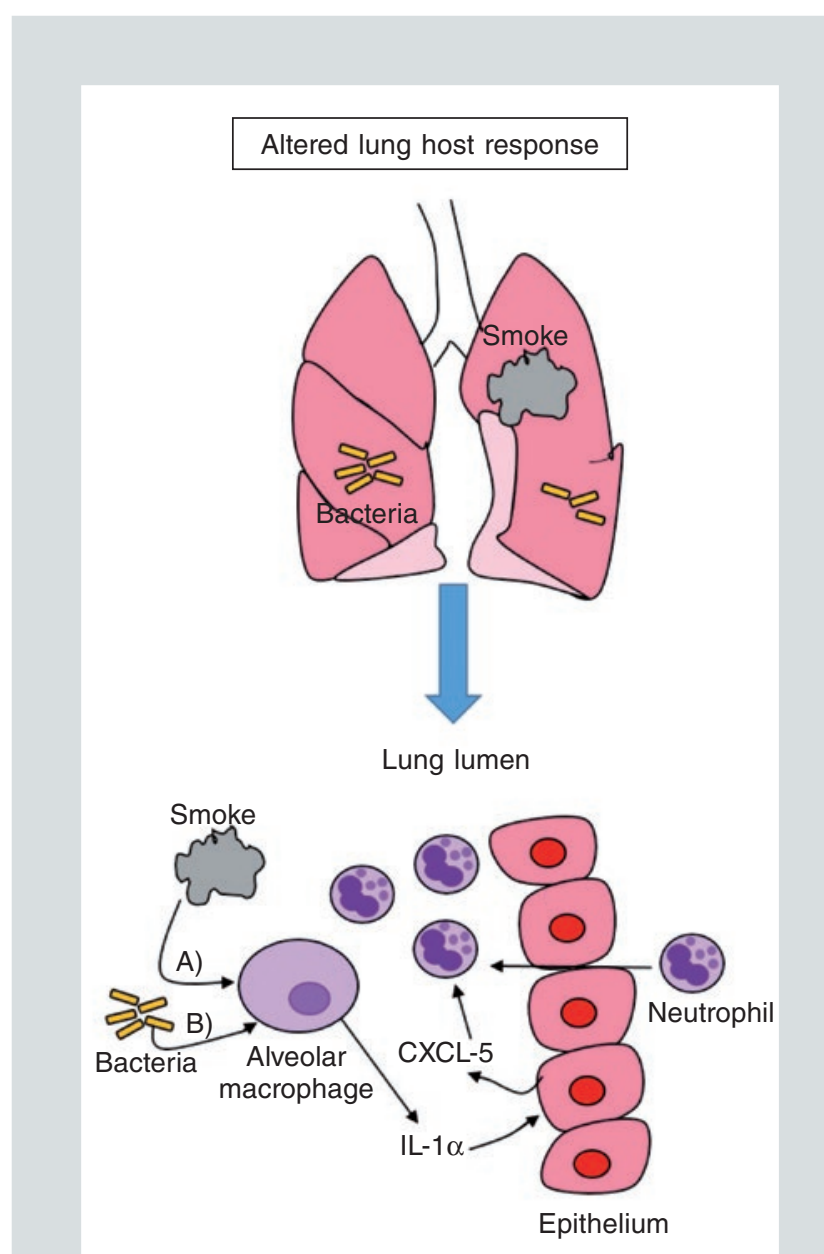

Figure 4. Cigarette smoke exposure alters the lung host response to bacteria. $\mathbf{A}$ : cigarette smoke induces alveolar macrophages to produce interleukin (IL)- $1 \alpha$, which acts on the epithelium to recruit neutrophils via chemokine (CXC-motif) ligand (CXCL)-5. B: bacterial stimulation further amplifies the IL-1 $\alpha$ response in alveolar macrophages, resulting in exacerbated neutrophilia associated with lung damage.

bacterial colonization. Enhanced bacterial virulence in smokers may also play a role. The higher risk of bacterial dissemination from the URT of smokers is reflected in a higher proportion of smokers with pathogenic bacterial lung colonization compared to non-smokers. Impaired lung mucociliary clearance and attenuated $\mathrm{AM}$ responses also contribute to lung colonization. Furthermore, smoking alters the lung host response to bacteria, even 
in asymptomatic smokers, to increase inflammation. This altered host response has consequences for the pathogenesis of COPD. Cigarette smoke primes the lungs towards IL-1 $\alpha$-dependent excessive neutrophilia. We speculate that repeated lung bacterial stimulation in smokers leads to lung damage, decline in lung function, and potentially the development of COPD. Notably, this process may also underlie acute bacterial exacerbations of COPD. Further understanding of how cigarette smoke impacts the URT host response is warranted. Control and containment of bacterial colonization, the first step of disease, is an attractive therapeutic goal that can reduce subsequent steps of disease in smokers and COPD patients.

\section{CONFLICT OF INTEREST}

\author{
Dr. Martin R. Stampfli reports grants from \\ Canadian Institutes of Health Research, grants \\ from Hoffmann-La Roche, during the conduct \\ of the study; personal fees from AstraZeneca, \\ personal fees from Boehringer Ingelheim, out- \\ side the submitted work. Dr. Pamela Shen has \\ nothing to disclose.
}

\section{REFERENCES}

1. National Center for Chronic Disease Prevention and Health Promotion (US) Office on Smoking and Health. The Health Consequences of Smoking-50 Years of Progress: A Report of the Surgeon General. 2014.

2. Stämpfli MR, Anderson GP. How cigarette smoke skews immune responses to promote infection, lung disease and cancer. Nat Rev Immunol. 2009; 9:377-84.

3. Almirall J, González CA, Balanzó X, et al. Proportion of community-acquired pneumonia cases attributable to tobacco smoking. Chest. 1999;116:375-9.

4. Kolappan C, Gopi PG. Tobacco smoking and pulmonary tuberculosis. Thorax. 2002;57:964-6.

5. Straus WL, Plouffe JF, File TMJ et al. Risk factors for domestic acquisition of legionnaires disease. Ohio legionnaires Disease Group. Arch Intern Med. 1996;156:1685-92.

6. Fischer M, Hedberg K, Cardosi P et al. Tobacco smoke as a risk factor for meningococcal disease. Pediatr Infect Dis J. 1997;16:979-83.
7. Murphy TF. Otitis media, bacterial colonization, and the smoking parent. Clin Infect Dis. 2006;42:904-6.

8. Nuorti JP, Butler JC, Farley MM et al. Cigarette smoking and invasive pneumococcal disease. Active Bacterial Core Surveillance Team. N Engl J Med. 2000;342:681-9.

9. Arbes SJ, Agústsdóttir H, Slade GD. Environmental tobacco smoke and periodontal disease in the United States. Am J Public Health. 2001;91:253-7.

10. Bello S, Menéndez R, Torres A et al. Tobacco Smoking Increases the Risk for Death From Pneumococcal Pneumonia. Chest. 2014;1029-37.

11. Almirall J, Blanquer J, Bello S. Community-acquired pneumonia among smokers. Arch Bronconeumol. 2014;50:250-4.

12. Human Microbiome Project Consortium T. Structure, function and diversity of the healthy human microbiome. Nature. 2012;486:207-14.

13. Bogaert D, De Groot R, Hermans PW. Streptococcus pneumoniae colonisation: the key to pneumococcal disease. Lancet Infect Dis. 2004;4:144-54.

14. Brook I, Gober AE. Recovery of potential pathogens and interfering bacteria in the nasopharynx of smokers and nonsmokers. Chest. 2005;127:2072-5.

15. Ramakrishnan VR, Feaze LM, Gitomer SA et al. The microbiome of the middle meatus in healthy adults. PLoS One. 2013;8:e85507.

16. Charlson ES, Chen J, Custers-Allen R et al. Disordered microbial communities in the upper respiratory tract of cigarette smokers. PLoS One. 2010; 5:e15216.

17. Cardozo DM, Nascimento-Carvalho CM, Andrade A-LSS et al. Prevalence and risk factors for nasopharyngeal carriage of Streptococcus pneumoniae among adolescents. J Med Microbiol. 2008;57:185-9.

18. Brook I, Gober AE. Effect of smoking cessation on the microbial flora. Arch Otolaryngol Head Neck Surg. 2007;133:135-8.

19. Ding T, Schloss PD. Dynamics and associations of microbial community types across the human body. Nature. 2014;509:357-60.

20. Faust K, Sathirapongsasuti JF, Izard J et al. Microbial co-occurrence relationships in the human microbiome. PLoS Comput Biol. 2012;8:e1002606.

21. Berger G, Bitterman R, Azzam ZS. The Human Microbiota: The Rise of an "Empire." Rambam Maimonides Med J. 2015;6:e0018.

22. Laufer AS, Metlay JP, Gent JF et al. Microbial communities of the upper respiratory tract and otitis media in children. MBio. 2011;2:e00245-10.

23. Cremers AJ, Zomer AL, Gritzfeld JF et al. The adult nasopharyngeal microbiome as a determinant of pneumococcal acquisition. Microbiome. 2014;2:44.

24. Zhang N, van Crombruggen $\mathrm{K}$, Gevaert $\mathrm{E}$ et al. Barrier function of the nasal mucosa in health and type-2 biased airway diseases. Allergy. 2016;71:295-307.

25. Schneeberger EE, Lynch RD. The tight junction: a multifunctional complex Am J Physiol Cell Physiol. 2004;286:C1213-28.

26. Heijink IH, Brandenburg SM, Postma DS et al. Cigarette smoke impairs airway epithelial barrier function and cell-cell contact recovery. Eur Respir J. 2012;39:419-28.

27. Sleigh MA, Blake JR, Llron N. The Propulsion of Mucus by Cilia. Am Rev Respir Dis.1988;137:726-41.

28. Schipper NG, Verhoef JC, Merkus FW. The nasal mucociliary clearance: relevance to nasal drug delivery. Pharm Res.1991;8:807-14.

29. Saetta M, Turato G, Baraldo S et al. Goblet cell hyperplasia and epithelial inflammation in peripheral airways of smokers with both symptoms of chronic bronchitis and chronic airflow limitation. Am J Respir Crit Care Med. 2000;161:1016-21.

30. Allinson JP, Hardy R, Donaldson GC, et al. The Presence of Chronic Mucus Hypersecretion Across Adult Life in Relation to COPD Development. Am J Respir Crit Care Med 2016;193:662-72.

31. Clunes LA., Davies CM, Coakley RD et al. Cigarette smoke exposure induces CFTR internalization and insolubility, leading to airway surface liquid dehydration. FASEB J. 2012;26:533-45.

32. Simet SM, Sisson JH, Pavlik JA et al. Long-term cigarette smoke exposure in a mouse model of ciliated epithelial cell function. Am J Respir Cell Mol Biol. 2010;43:635-40.

33. Pagliuca G, Rosato C, Martellucci S et al. Cytologic and Functional Alterations of Nasal Mucosa in Smokers: Temporary or Permanent Damage? Otolaryngol -- Head Neck Surg. 2015;152:740-5. 
34. Livraghi-Butrico A, Kelly EJ, Klem ER et al. Mucus clearance, MyD88-dependent and MyD88-independent immunity modulate lung susceptibility to spontaneous bacterial infection and inflammation. Mucosal Immunol. 2012;5:397-408.

35. Shen P, Morissette MC, Vanderstocken G et al. Cigarette smoke attenuates the nasal host response to Streptococcus pneumoniae and predisposes to invasive pneumococcal disease in mice. Infect Immun. 2016;84:1536-47.

36. Effros RM, Mason GR, Mena I. 99mTc-DTPA aerosol deposition and clearance in COPD, interstitial disease, and smokers. J Thorac Imaging. 1986;1:54-60.

37. Rayner CFJ, Jackson AD, Rutman A et al. Interaction of pneumolysin-sufficient and -deficient isogenic variants of Streptococcus pneumoniae with human respiratory mucosa. Infect Immun. 1995;63:4427.

38. Clarke TB, Francella N, Huegel A et al. Invasive bacterial pathogens exploit TLR-mediated downregulation of tight junction components to facilitate translocation across the epithelium. Cell Host Microbe. 2011;9:404-14.

39. Piatti G, Gazzola T, Allegra L. Bacterial adherence in smokers and nonsmokers. Pharmacol Res. 1997;36:481-4.

40. Raman AS, Swinburne AJ, Fedullo AJ. Pneumococcal adherence to the buccal epithelial cells of cigarette smokers. Chest. 1983;83:23-7.

41. Zhang JR, Mostov KE, Lamm ME et al. The polymeric immunoglobulin receptor translocates pneumococci across human nasopharyngeal epithelial cells. Cell. 2000;102:827-37.

42. Cundell DR, Gerard NP, Gerard C et al. Streptococcus pneumoniae anchor to activated human cells by the receptor for platelet-activating factor. Nature. 1995;377:435-8.

43. Grigg J, Walters H, Sohal SS et al. Cigarette smoke and platelet-activating factor receptor dependent adhesion of Streptococcus pneumoniae to lower airway cells. Thorax. 2012;67:908-13.

44. Grigg J. The platelet activating factor receptor: a new anti-infective target in respiratory disease? Thorax. 2012;67:840-1.

45. Barbier M, Oliver A, Rao J et al. Novel phosphorylcholine-containing protein of Pseudomonas aeruginosa chronic infection isolates interacts with airway epithelial cells. JID. 2008;197:465-73.

46. Brown AO, Mann B, Gao G et al. Streptococcus pneumoniae Translocates into the Myocardium and Forms Unique Microlesions That Disrupt Cardiac Function. PLoS Pathog. 2014;10:e1004383.

47. Ring A, Weiser JN, Tuomanen EI. Pneumococcal trafficking across the blood-brain barrier molecular analysis of a novel bidirectional pathway. J Clin Invest. 1998;102:347-60.

48. Ohlmeier S, Mazur W, Linja-Aho A et al. Sputum proteomics identifies elevated PIGR levels in smokers and mild-to-moderate COPD. J Proteome Res. 2012;11:599-608.

49. Gohy ST, Detry BR, Lecocq M et al. Polymeric immunoglobulin receptor down-regulation in chronic obstructive pulmonary disease: Persistence in the cultured epithelium and role of transforming growth factor- $\beta$. Am J Respir Crit Care Med. 2014;190:509-21.

50. Charlson ES, Bittinger K, Haas AR et al. Topographical continuity of bacterial populations in the healthy human respiratory tract. Am J Respir Crit Care Med. 2011;184:957-63.

51. Lee C, Middaugh N, Howie S et al. Association of secondhand smoke exposure with pediatric invasive bacterial disease and bacterial carriage: A systematic review and meta-analysis. PLoS Med. 2010;7:1-14.

52. González-Del Vecchio M, Bunsow E, Sánchez-Carrillo C et al. Occult bloodstream infections in adults: A “benign” entity. Am J Emerg Med. 2014;32:966-71.

53. Zhang Z, Clarke TB, Weiser JN. Cellular effectors mediating Th17-dependent clearance of pneumococcal colonization in mice. J Clin Invest. 2009; 119:1899-909.

54. Matthias KA, Roche AM, Standish AJ et al. Neutrophil-toxin interactions promote antigen delivery and mucosal clearance of Streptococcus pneumoniae. J Immunol. 2008;180:6246-54.
55. Munoz-Elias EJ, Marcano J et al. Isolation of Streptococcus pneumoniae biofilm mutants and their characterization during nasopharyngeal colonization. Infect Immun. 2008;76:5049-61.

56. Hall-Stoodley L, Hu FZ, Gieseke A et al. Direct detection of bacterial biofilms on the middle-ear mucosa of children with chronic otitis media. JAMA. 2006;296:202-11.

57. Sanchez CJ, Shivshankar P, Stol K et al. The pneumococcal serine-rich repeat protein is an intra-species bacterial adhesin that promotes bacterial aggregation in vivo and in biofilms. PLoS Pathog. 2010;6:e1001044.

58. Moscoso M, Garcia E, Lopez R. Pneumococcal biofilms. Int Microbiol. 2009;12:77-85.

59. Antunes MB, Chi JJ, Liu Z et al. Molecular basis of tobacco-induced bacterial biofilms: an in vitro study. Otolaryngol Head Neck Surg. 2012;147: 876-84.

60. Bagaitkar J, Williams LR, Renaud DE et al. Tobacco-induced alterations to Porphyromonas gingivalis-host interactions. Env Microbiol. 2009;11: 1242-53.

61. Kulkarni R, Antala S, Wang A et al. Cigarette smoke increases Staphylococcus aureus biofilm formation via oxidative stress. Infect Immun. 2012;80: 3804-11.

62. Mutepe ND, Cockeran R, Steel HC et al. Effects of cigarette smoke condensate on pneumococcal biofilm formation and pneumolysin. Eur Respir J 2012;41:392-5.

63. Soler N, Ewig S, Torres A et al. Airway inflammation and bronchial microbial patterns in patients with stable chronic obstructive pulmonary disease. Eur Respir J. 1999;14:1015-22.

64. Hogg JC, Chu F, Utokaparch S, Wet al. The nature of small-airway obstruction in chronic obstructive pulmonary disease. N Engl J Med. 2004;350: 2645-53.

65. Berenson CS, Wrona CT, Grove LJ et al. Impaired alveolar macrophage response to Haemophilus antigens in chronic obstructive lung disease. Am J Respir Crit Care Med. 2006;174:31-40.

66. Berenson CS, Garlipp MA, Grove LJ et al. Impaired phagocytosis of nontypeable Haemophilus influenzae by human alveolar macrophages in chronic obstructive pulmonary disease. J Infect Dis. 2006;194:1375-84.

67. Gaschler GJ, Skrtic M, Zavitz CC et al. Bacteria challenge in smoke-exposed mice exacerbates inflammation and skews the inflammatory profile. Am J Respir Crit Care Med. 2009;179:666-75.

68. Nikota JK, Shen P, Morissette MC et al. Cigarette Smoke Primes the Pulmonary Environment to IL-1 /CXCR-2-Dependent Nontypeable Haemophilus influenzae-Exacerbated Neutrophilia in Mice. J Immunol. 2014;193 3134-45.

69. Sethi S, Murphy TF. Bacterial infection in chronic obstructive pulmonary disease in 2000: a state-of-the-art review. Clin Microbiol Rev. 2011;14 336-63.

70. Salvi S. Tobacco smoking and environmental risk factors for chronic obstructive pulmonary disease. Clin Chest Med. 2014;35:17-27.

71. Mulhall P, Criner G. Non-pharmacological treatments for COPD. Respirology. 2016;21:791-809.

72. Gaschler GJ, Zavitz CC, Bauer CM et al. Cigarette smoke exposure attenuates cytokine production by mouse alveolar macrophages. Am J Respir Cell Mol Biol. 2008;38:218-26.

73. Botelho FM, Bauer CM, Finch D et al. IL-1alpha/IL-1R1 expression in chronic obstructive pulmonary disease and mechanistic relevance to smokeinduced neutrophilia in mice. PLoS One. 2011;6:e28457.

74. Dinarello CA. Immunological and inflammatory functions of the interleukin-1 family. Annu Rev Immunol. 2009;27:519-50.

75. Morissette MC, Shen P, Thayaparan D et al. Disruption of pulmonary lipid homeostasis drives cigarette smoke-induced lung inflammation in mice. Eur Respir J. 2015;46:1451-60. 